\title{
Spanish Chestnut Cultivars
}

Santiago Pereira-Lorenzo ${ }^{1}$, Ana M. Ramos-Cabrer,
Belén Díaz-Hernández, Javier Ascasíbar-Errasti, and Federico Sau

Departamento de Producción Vegetal, Universidad de Santiago de Compostela, Campus de Lugo, 27002 Lugo, Spain

\section{Marta Ciordia-Ara}

SERIDA, Consejería de Medio Rural y Pesca, Principado de Asturias, Apt. 13, 33300, Villaviciosa, Asturias, Spain

Additional index words. germplasm resources, variability, local cultivars

Abstract. Chestnut (Castanea sativa Mill.) is an important crop in Spain. This inventory of chestnut cultivars complements previous studies. We have located 152 chestnut cultivars in 131 municipalities covering 108.6 ha, with 72 new cultivars in addition to the 80 previously found. Fewer than $50 \%$ of these cultivars are extensively cultivated. Chestnuts in Spain are grown from sea level to $1100 \mathrm{~m}$, but are more frequent between 200 and $800 \mathrm{~m}$ on northern-facing slopes. Most of the chestnuts are harvested from 25 Oct. to 10 Nov.

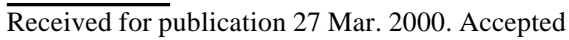
for publication 3 Aug. 2000. The support of INIA (Programa Nacional de Recursos Genéticos) and FEDER (Desarrollo Integral del Castaño en Asturias) is gratefully acknowledged. We also acknowledge the editorial assistance of Sandra Anagnostakis. The cost of publishing this paper was defrayed in part by the payment of page charges. Under postal regulations, this paper therefore must be hereby marked advertisement solely to indicate this fact.

${ }^{1}$ To whom requests for reprints should be addressed. E-mail address: spereira@lugo.usc.es
Most of the chestnuts grown in Spain are found in the northwestern part of the country in the regions of Galicia and Asturias (Fig. 1). Previous papers have listed cultivars in Galicia (Fernández and Pereira, 1994; Pereira-Lorenzo and Fernández-López, 1997) and Asturias (Fernández-Lamuño, 1984). Our long-term goal has been to establish a national germplasm bank of the genus Castanea.

In País Vasco and Navarra, chestnut orchards have been destroyed by chestnut blight disease, caused by the fungus Cryphonectria parasitica (Murr.) Barr. This was surely introduced with Asian chestnut seeds in 1914 and 1940 (Elorrieta, 1949), although it was first reported in 1943 (Colinas and Uscuplic, 1999). In Cataluña, coppicing is predominant and orchards have been abandoned for a long time. In this work, we present the results of sampling the five most important chestnut-producing regions, viz., eastern Galicia, (Pereira-Lorenzo and Fernández-López, 1997; Pereira-Lorenzo et al., 1996a, 1996b), Andalucía, Asturias, Extremadura, and Castilla-León (Fig. 1).

\section{Materials and Methods}

Locating trees. Trees were located by traveling throughout the chestnut-growing areas described by Elorrieta (1949) and asking chestnut growers to identify representative trees of each cultivar. Each tree of a specific cultivar was marked for later sampling.

Data collection. For each tree, we recorded the altitude with an altimeter and the solar orientation of the planting site with a compass. During autumn, nut samples were collected for morphological characterization, and the harvest time was recorded. We compiled data for each region, with information on the cultivar, municipality, elevation, aspect, and time of harvest. Only those cultivars found in more than four municipalities (except for those in the Andalucía and Extremadura regions, where few trees were found) were reported on the map of cultivar distribution (Fig. 1), and chestnut area was taken from Ministerio de Agricultura, Pesca y Alimentación (1997).

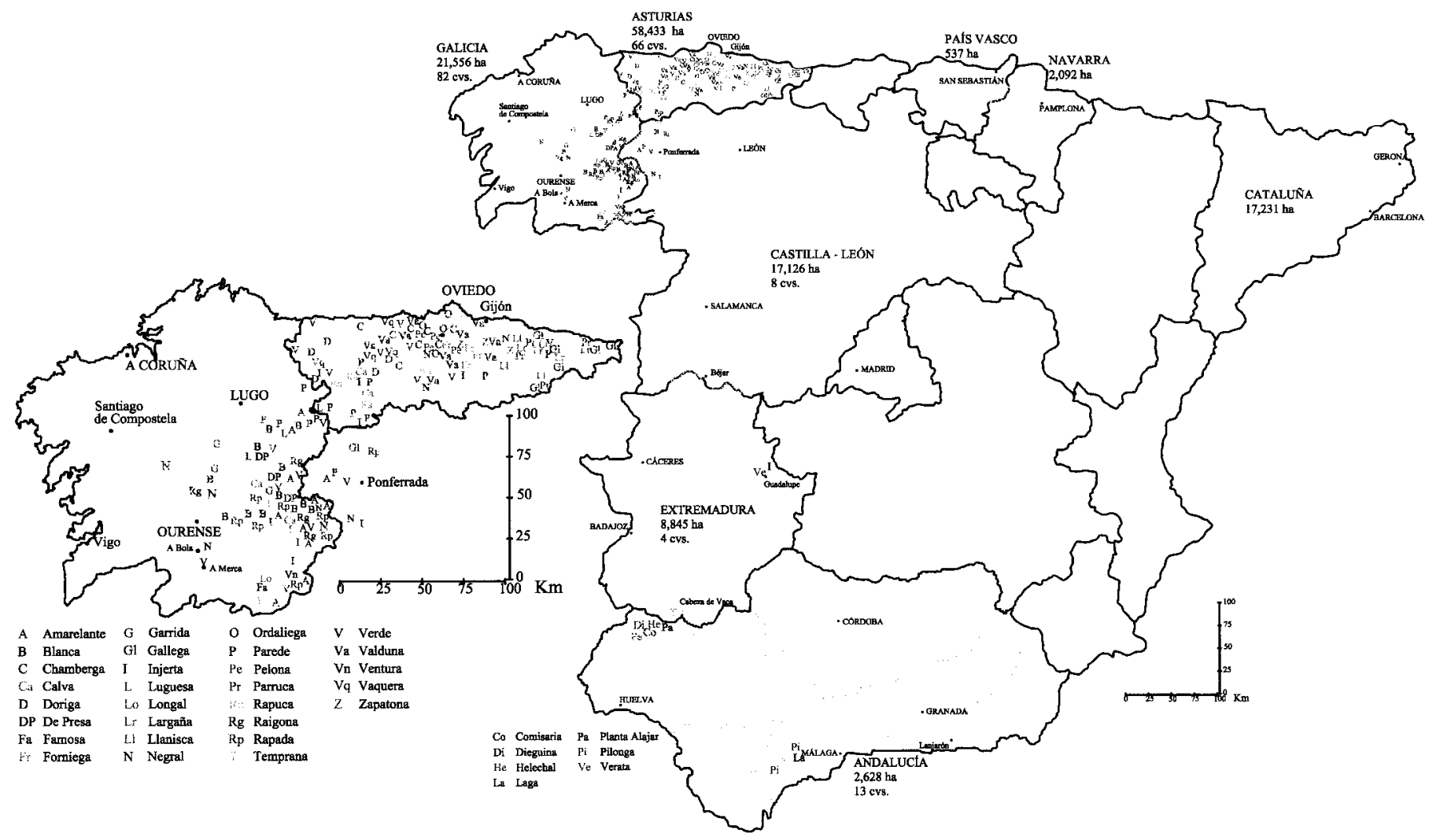

Fig. 1. Distribution of chestnut (Castanea sativa Mill.) cultivars in Spain 
Table 1. Chestnut cultivars found in Huelva and Málaga provinces, Andalucía.

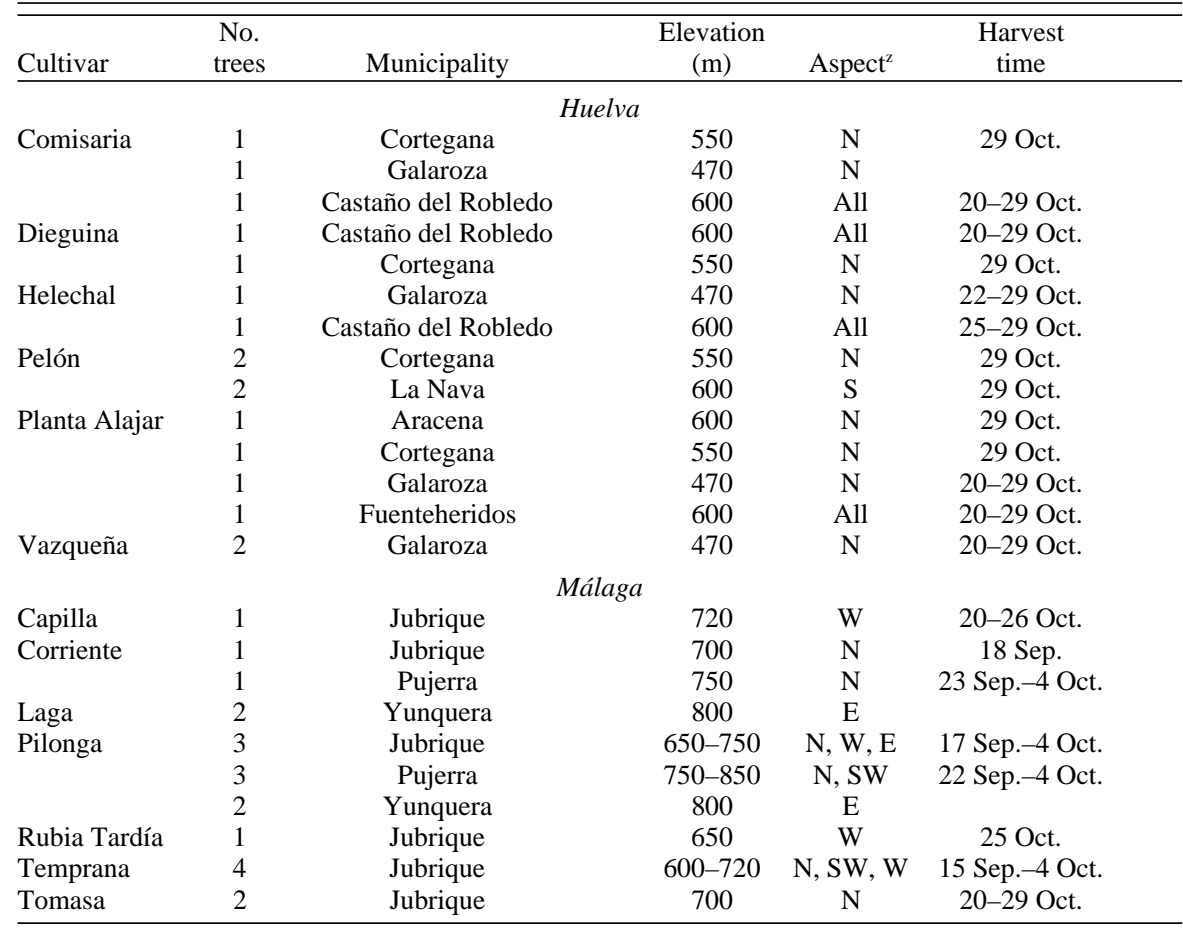

${ }^{\mathrm{z}} \mathrm{N}, \mathrm{All}, \mathrm{S}, \mathrm{W}, \mathrm{E}$, and SW = north-facing, no predominant aspect, south-facing, west-facing, east-facing, and southwest-facing slopes, respectively.
Orchard areas in the map are marked with letters corresponding to the main cultivars.

\section{Results and Discussion}

We examined 65 municipalities in Galicia (Fernández and Pereira, 1994), nine in Andalucía, 49 in Asturias, six in Castilla-León, and two in Extremadura (Fig. 1 and Tables 15). Only municipalities with cultivars that could be positively identified are presented. In southern Castilla-León, cultivars could not be identified by the growers, some orchards were abandoned, and thus were not used in this study. In Extremadura, some growers graft selected cultivars on seedling chestnuts. In southern Andalucia, chestnuts are grafted in Huelva and Málaga, but not in Granada.

Cultivars found. We found 66 cultivars in Asturias, 13 in Andalucía, eight in CastillaLeón, four in Extremadura, and four in a part of Galicia not previously studied. Of the cultivars reported by Fernández-Lamuño (1984) in Asturias, and by Fernández and Pereira (1994) in Galicia, we found only 'Temprana' in Andalucía. Tests are in progress to determine if the identification was correct. In Asturias, we found 43 new cultivars, in addition to the 14 reported by Fernández-Lamuño (1984). Four of these were found by Fernández and
Table 2. Chestnut cultivars found in Asturias.

\begin{tabular}{|c|c|c|c|c|c|}
\hline Cultivar & $\begin{array}{l}\text { No. } \\
\text { trees }\end{array}$ & Municipality & $\begin{array}{c}\text { Elevation } \\
(\mathrm{m})\end{array}$ & Aspect $^{2}$ & $\begin{array}{c}\text { Harvest } \\
\text { time }\end{array}$ \\
\hline \multirow[t]{2}{*}{ Argua } & 1 & Colunga & 410 & $\mathrm{~S}$ & 23 Nov. \\
\hline & 1 & Piloña & 450 & $\mathrm{~S}$ & 5 Nov. \\
\hline Baragaña & 2 & Santo Adriano & 600 & $\mathrm{E}$ & 29 Oct. \\
\hline Boroñona & 1 & Mieres & 450 & SE & 4 Nov. \\
\hline Brullina & 1 & Llanes & 150 & All & 2 Nov. \\
\hline \multirow[t]{2}{*}{ Calva } & 2 & Cangas del Narcea & 450 & $\mathrm{E}$ & $23-$ \\
\hline & 1 & Pola de Allande & 600 & All & 26 Oct. \\
\hline Cañuela & 1 & Langreo & 410 & $\mathrm{~W}$ & 28 Oct. \\
\hline Caranquexa & 1 & Quirós & 340 & NW & 27 Oct. \\
\hline Carreña & 1 & Candamo & 50 & $\mathrm{NE}$ & 26 Oct. \\
\hline Cerbana & 1 & Langreo & 350 & $\mathrm{E}$ & 27 Oct. \\
\hline Cofina & 1 & Llanes & 500 & & 2 Nov. \\
\hline Colunga & 1 & Llanes & 500 & & 2 Nov. \\
\hline \multirow[t]{3}{*}{ Crespa } & 1 & Laviana & 450 & $\mathrm{E}$ & 28 Oct. \\
\hline & 2 & Piloña & $300-420$ & W & 28 Oct. \\
\hline & 1 & Sobrescobio & 400 & $\mathrm{~W}$ & 26 Oct. \\
\hline Cruz & 1 & Piloña & 450 & $\mathrm{~S}$ & 5 Nov. \\
\hline \multirow[t]{7}{*}{ Chamberga } & 3 & Belmonte de Miranda & $300-350$ & E, W, NW & 27 Oct. \\
\hline & 1 & Candamo & 50 & $\mathrm{NE}$ & 26 Oct. \\
\hline & 3 & Grado & $275-520$ & $\mathrm{~W}, \mathrm{NW}$ & 26 Oct. \\
\hline & 3 & Las Regueras & $60-120$ & $\mathrm{~W}, \mathrm{~S}$ & 26 Oct. \\
\hline & 1 & Pravia & 350 & W & 4 Nov. \\
\hline & 3 & Salas & $100-480$ & $\mathrm{~N}, \mathrm{E}$ & $\begin{array}{l}19 \text { Oct.- } \\
4 \text { Nov. }\end{array}$ \\
\hline & 1 & Valdés & 120 & $\mathrm{~S}$ & \\
\hline Chancloya & 1 & Belmonte de Miranda & 300 & $\mathrm{E}$ & 27 Oct. \\
\hline \multirow[t]{5}{*}{ Doriga } & 1 & Boal & 430 & $\mathrm{E}$ & 25 Oct. \\
\hline & 1 & Ibias & 600 & $\mathrm{E}$ & 23 Oct. \\
\hline & 1 & Pesoz & 450 & $\mathrm{~N}$ & 25 Oct. \\
\hline & 5 & Pola de Allande & $420-690$ & $\mathrm{~N}, \mathrm{~S}, \mathrm{~W}$ & $\begin{array}{l}23 \text { Oct.- } \\
4 \text { Nov. }\end{array}$ \\
\hline & 2 & Tineo & $300-400$ & W, SW & 27 Oct. \\
\hline Escamplero & 1 & Valdés & 340 & $\mathrm{~S}$ & 7 Nov. \\
\hline Fano & 1 & Quirós & 340 & NW & 27 Oct. \\
\hline \multirow[t]{2}{*}{ Feltrona } & 1 & Quirós & 340 & NW & 27 Oct. \\
\hline & 1 & Santo Adriano & 240 & $\mathrm{~N}$ & 29 Oct. \\
\hline \multirow[t]{3}{*}{ Forniega } & 1 & Aller & 629 & $\mathrm{NE}$ & 2 Nov. \\
\hline & 1 & Langreo & 410 & W & 28 Oct. \\
\hline & 2 & Mieres & $300-450$ & $\mathrm{~W}, \mathrm{SE}$ & 2 Nov. \\
\hline
\end{tabular}

Table 2. Continued.

\begin{tabular}{|c|c|c|c|c|c|}
\hline Cultivar & $\begin{array}{l}\text { No. } \\
\text { trees }\end{array}$ & Municipality & $\begin{array}{l}\text { Elevation } \\
(\mathrm{m})\end{array}$ & Aspect $^{z}$ & $\begin{array}{c}\text { Harvest } \\
\text { time }\end{array}$ \\
\hline & 2 & Oviedo & 280 & $\mathrm{~N}, \mathrm{E}$ & 29 Oct. \\
\hline & 1 & $\begin{array}{c}\text { San Martín del } \\
\text { Rey Aurelio }\end{array}$ & 560 & $\mathrm{E}$ & \\
\hline \multirow[t]{10}{*}{ Galega } & 3 & Amieva & $80-120$ & All & 28 Oct. \\
\hline & 1 & Boal & 290 & All & 25 Oct. \\
\hline & 1 & Cabrales & 50 & All & 2 Nov. \\
\hline & 2 & Cangas de Onís & 80 & $\mathrm{~W}, \mathrm{E}$ & $\begin{array}{l}28 \text { Oct.- } \\
2 \text { Nov. }\end{array}$ \\
\hline & 1 & Ibias & 600 & $\mathrm{E}$ & 24 Oct. \\
\hline & 1 & Parres & 210 & NW & 28 Oct. \\
\hline & 2 & Peñamellera alta & 10 & $\mathrm{~N}$ & 2 Nov. \\
\hline & 1 & Pesoz & 240 & & 25 Oct. \\
\hline & 1 & Ponga & 200 & S & 28 Oct. \\
\hline & 1 & Oscos & 600 & S & 26 Oct. \\
\hline \multirow[t]{2}{*}{ De la Grúa } & 1 & Parres & 240 & W & 28 Oct. \\
\hline & 4 & Villaviciosa & $100-180$ & $\mathrm{~N}, \mathrm{~S}$ & $\begin{array}{l}19 \text { Oct.- } \\
12 \text { Nov. }\end{array}$ \\
\hline \multirow[t]{4}{*}{ Inxerta } & 2 & Aller & $530-700$ & $\mathrm{~N}$ & 2 Nov. \\
\hline & 1 & Cangas del Narcea & 830 & W & 27 Oct. \\
\hline & 3 & Degaña & $750-900$ & $\mathrm{~W}$ & 27 Oct. \\
\hline & 2 & Ibias & $840-880$ & S, E & 22 Oct. \\
\hline \multirow[t]{4}{*}{ Largaña } & 1 & Cabrales & 50 & All & 2 Nov. \\
\hline & 5 & Cangas de Onís & $10-80$ & All & $\begin{array}{l}28 \text { Oct.- } \\
2 \text { Nov. }\end{array}$ \\
\hline & 1 & Parres & 240 & $\mathrm{~W}$ & 28 Oct. \\
\hline & 1 & Peñamellera alta & 10 & $\mathrm{~N}$ & 2 Nov. \\
\hline \multirow[t]{3}{*}{ Leinova } & 1 & Oscos & 630 & $\mathrm{E}$ & 26 Oct. \\
\hline & 1 & Tapia de Casariego & 80 & $\mathrm{E}$ & 25 Oct. \\
\hline & 1 & Taramundi & 300 & $\mathrm{~N}$ & 26 Oct. \\
\hline Loura & 1 & Oscos & 630 & $\mathrm{E}$ & 26 Oct. \\
\hline \multirow[t]{4}{*}{ Llanisca } & 1 & Parres & 240 & W & 28 Oct. \\
\hline & 5 & Piloña & $270-420$ & $\mathrm{~W}, \mathrm{E}, \mathrm{N}$ & 28 Oct. \\
\hline & 2 & Ponga & $200-615$ & S, SW & 28 Oct. \\
\hline & 1 & Sobrescobio & 400 & W & \\
\hline Mariana & 1 & Villaviciosa & 170 & $\mathrm{~N}$ & 19 Oct. \\
\hline Marimoeche & 2 & Pola de Allande & $550-660$ & $\mathrm{E}$ & 23 Oct. \\
\hline \multirow[t]{2}{*}{ Marina } & 1 & Laviana & 570 & $\mathrm{E}$ & 4 Nov. \\
\hline & 1 & Proaza & 300 & W & 29 Oct. \\
\hline Mediana & 1 & Teverga & 600 & $\mathrm{E}$ & 28 Oct. \\
\hline Miguelina & 1 & Pravia & 300 & $\mathrm{NE}$ & 4 Nov. \\
\hline
\end{tabular}


Pereira (1994) in Galicia, and five in both Galicia and Asturias.

In Castilla-León, all cultivars found were reported previously in Asturias as 'Rapega' (or 'Rapuca') and 'Gallego' (FernándezLamuño, 1984); in Galicia as 'Inxerta', 'Marelo', and 'Rapada' (Fernández and Pereira, 1994); and in both Asturias and Galicia as 'Negral', 'Parede', and 'Verdello'. In Extremadura, only 'Verata' was a new name, because 'Temprana' was reported in Andalucía and Galicia, 'Tardía' in Asturias and Galicia ('Serodia' in the local dialect), and 'Inxerta' in
Asturias, Galicia, and Castilla-León. In the new area surveyed in Galicia, only 'De Fecha' and 'Cruzá' were not previously reported (Fernández and Pereira, 1994).

Some of the cultivars are distributed across a range of $>100 \mathrm{~km}$ (Fig. 1). For example, 'Inxerta' is known from Galicia and Asturias to Andalucía, and 'Parede' is cultivated in Galicia, Asturias, and Castilla-León. This wide distribution suggests that the domestication process in the Middle Ages was based on grafting of the most profitable cultivars. In contrast, six cultivars in Andalucía were found only in one municipality (Table 1), while in Asturias there were 30 cultivars in one location (Table 2).

Galicia had the largest number of cultivars (Fernández and Pereira, 1994) (16 more than Asturias) although it has less than half the area of Asturial (Fig. 1) because of the transformation of the orchards in Asturias into forest management for timber in the 1950s. In Extremadura, growers have selected fewer cultivars than in Andalucía. In Andalucía, management of chestnut for timber was important, but an increase in the price of
Table 2. Continued.

\begin{tabular}{|c|c|c|c|c|c|}
\hline Cultivar & $\begin{array}{l}\text { No. } \\
\text { trees }\end{array}$ & Municipality & $\begin{array}{c}\text { Elevation } \\
(\mathrm{m})\end{array}$ & Aspect $^{2}$ & $\begin{array}{c}\text { Harvest } \\
\text { time }\end{array}$ \\
\hline & 3 & Salas & $100-480$ & $\mathrm{~N}, \mathrm{E}$ & $\begin{array}{l}19 \text { Oct.- } \\
4 \text { Nov. }\end{array}$ \\
\hline Montesín & 1 & Villaviciosa & 250 & & 19 Oct. \\
\hline Mourisco & 2 & Pola de Allande & $550-600$ & W & 26 Oct. \\
\hline \multirow[t]{2}{*}{ Nargana } & 1 & Llanes & 500 & & 2 Nov. \\
\hline & 4 & Peñamellera alta & $100-380$ & All & 2 Nov. \\
\hline \multirow[t]{2}{*}{ Navexa } & 1 & Belmonte de Miranda & 330 & NW & 27 Nov. \\
\hline & 3 & Tineo & $300-400$ & All & $\begin{array}{c}24- \\
27 \text { Oct. }\end{array}$ \\
\hline \multirow[t]{3}{*}{ Negral } & 1 & Santo Adriano & 720 & & 4 Nov. \\
\hline & 1 & Teverga & 700 & $\mathrm{~N}$ & 28 Oct. \\
\hline & 1 & Villaviciosa & 100 & NW & 19 Oct. \\
\hline \multirow[t]{4}{*}{ Ordaliega } & 2 & Corvera & 150 & All & 18 Nov. \\
\hline & 1 & Illas & 340 & $\mathrm{~W}$ & \\
\hline & 2 & Las Regueras & 100 & & 26 Oct. \\
\hline & 2 & Pravia & $300-340$ & NE, S & 4 Nov. \\
\hline Padana & 1 & Pola de Allande & 550 & & 26 Oct. \\
\hline \multirow[t]{3}{*}{ Pagana } & 1 & Candamo & 50 & $\mathrm{NE}$ & \\
\hline & 1 & Cangas del Narcea & 440 & $\mathrm{E}$ & 23 Oct. \\
\hline & 1 & Villayón & 300 & W & \\
\hline Palaciana & 1 & Mieres & 300 & $\mathrm{~S}$ & 2 Nov. \\
\hline Panchina & 1 & Belmonte de Miranda & 350 & W & 27 Oct. \\
\hline \multirow[t]{10}{*}{ Parede } & 1 & Boal & 430 & $\mathrm{E}$ & 25 Oct. \\
\hline & 8 & Cangas del Narcea & $450-700$ & $\mathrm{~W}, \mathrm{E}, \mathrm{S}, \mathrm{N}$ & $22-$ \\
\hline & 1 & Castropol & 10 & $\mathrm{~N}$ & \\
\hline & 5 & Ibias & $300-780$ & $\mathrm{E}, \mathrm{S}$ & 23 Oct. \\
\hline & 1 & Pesoz & 220 & $\mathrm{~N}$ & 25 Oct. \\
\hline & 10 & Pola de Allande & $420-690$ & All & 22 Oct.- \\
\hline & 2 & Oscos & $580-600$ & All & 4 Nov. \\
\hline & 2 & Tapia de Casariego & $30-200$ & All & 25 Oct. \\
\hline & 2 & Taramundi & 300 & $\mathrm{~N}$ & 26 Oct. \\
\hline & 1 & Vegadeo & 270 & $\mathrm{E}$ & \\
\hline \multirow[t]{3}{*}{ Parruca } & 1 & Parres & 210 & NW & 28 Oct. \\
\hline & 4 & Piloña & 400 & $\mathrm{~N}, \mathrm{~S}$ & $\begin{array}{l}28 \text { Oct.- } \\
4 \text { Nov. }\end{array}$ \\
\hline & 1 & Ponga & 615 & SW & 28 Oct. \\
\hline Pelgo & 1 & Pola de Allande & 600 & W & 26 Oct. \\
\hline \multirow[t]{5}{*}{ Pelona } & 1 & Grado & 520 & W & 26 Oct. \\
\hline & 1 & Mieres & 300 & $\mathrm{~S}$ & 2 Nov. \\
\hline & 3 & Oviedo & $150-270$ & $\mathrm{E}, \mathrm{W}$ & 19 Oct.- \\
\hline & & & & & 29 Oct. \\
\hline & 2 & Santo Adriano & $600-650$ & $\mathrm{E}$ & $\begin{array}{c}29 \text { Oct.- } \\
4 \text { Nov. }\end{array}$ \\
\hline \multirow[t]{2}{*}{ Pelosa } & 1 & Candamo & 50 & $\mathrm{NE}$ & 26 Oct. \\
\hline & 1 & Cangas de Onís & 10 & $\mathrm{~N}$ & 28 Oct. \\
\hline \multirow[t]{3}{*}{ Ramoniega } & 1 & Bimenes & 350 & $\mathrm{~N}$ & \\
\hline & 2 & Siero & $200-400$ & $\mathrm{~N}, \mathrm{E}$ & 25 Oct.- \\
\hline & 1 & Sob & 400 & W & $\begin{array}{l}3 \text { Nov. } \\
26 \text { Oct. }\end{array}$ \\
\hline \multirow[t]{6}{*}{ Rapuca } & 5 & Cangas del Narcea & $440-600$ & $\mathrm{E}, \mathrm{N}, \mathrm{S}$ & $22-$ \\
\hline & & & & & 27 Oct. \\
\hline & 1 & Ibias & 560 & $\mathrm{~S}$ & 24 Oct. \\
\hline & 6 & Pola de Allande & $600-750$ & All & 24 Oct.- \\
\hline & 1 & Teverga & 700 & $\mathrm{~N}$ & $\begin{array}{l}4 \text { Nov. } \\
28 \text { Oct }\end{array}$ \\
\hline & 2 & Tineo & $350-440$ & All & 24 Oct. \\
\hline
\end{tabular}

Table 2. Continued.

\begin{tabular}{|c|c|c|c|c|c|}
\hline Cultivar & $\begin{array}{l}\text { No. } \\
\text { trees }\end{array}$ & Municipality & $\begin{array}{l}\text { Elevation } \\
\text { (m) }\end{array}$ & Aspect ${ }^{2}$ & $\begin{array}{c}\text { Harvest } \\
\text { time }\end{array}$ \\
\hline Ravexa & 1 & Pola de Allande & 420 & $\mathrm{~N}$ & \\
\hline \multirow[t]{3}{*}{ Roxa } & 1 & Laviana & 310 & & 26 Oct. \\
\hline & 1 & Piloña & 420 & W & 28 Oct. \\
\hline & 1 & Sobrescobio & 400 & W & 26 Oct. \\
\hline Roxina & 1 & Caravia Baja & 100 & $\mathrm{~N}$ & 2 Nov. \\
\hline \multirow[t]{2}{*}{ Rubia } & 1 & Pola de Allande & 420 & $\mathrm{~N}$ & 23 Oct. \\
\hline & 1 & Santo Adriano & 650 & $\mathrm{E}$ & \\
\hline Seronda & 1 & Proaza & 300 & W & 29 Oct. \\
\hline \multirow[t]{3}{*}{ Sevillana } & 2 & Caso & $530-660$ & $\mathrm{~S}$ & 26 Oct. \\
\hline & 1 & Piloña & 400 & $\mathrm{~S}$ & \\
\hline & 1 & Sobrescobio & 400 & W & 26 Oct. \\
\hline Tamón & 1 & Gijón & 300 & $\mathrm{E}$ & 3 Nov. \\
\hline Tixera & 1 & Proaza & 300 & W & 29 Oct. \\
\hline \multirow[t]{15}{*}{ Valduna } & 1 & Bimenes & 350 & $\mathrm{~N}$ & \\
\hline & 1 & Gijón & 30 & $\mathrm{~N}$ & 18 Nov. \\
\hline & 1 & Langreo & 350 & $\mathrm{E}$ & \\
\hline & 1 & Las Regueras & 210 & $\mathrm{~S}$ & 26 Oct. \\
\hline & 2 & Mieres & $300-430$ & $\mathrm{~S}$ & 2 Nov. \\
\hline & 4 & Oviedo & $150-280$ & $\mathrm{~N}, \mathrm{E}, \mathrm{W}$ & 19- \\
\hline & 1 & Pola de Allande & 690 & $\mathrm{~S}$ & $\begin{array}{l}29 \text { Oct. } \\
4 \text { Nov. }\end{array}$ \\
\hline & 1 & Pravia & 340 & $\mathrm{~S}$ & 4 Nov. \\
\hline & 1 & Salas & 130 & $\mathrm{~N}$ & 19 Oct. \\
\hline & 2 & Santo Adriano & $200-600$ & All & 29 Oct. \\
\hline & 6 & Siero & $200-500$ & All & 2 Nov. \\
\hline & 1 & Tapia de Casariego & 80 & $\mathrm{E}$ & 25 Oct. \\
\hline & 1 & Teverga & 600 & $\mathrm{E}$ & 28 Oct. \\
\hline & 2 & Tineo & $300-400$ & W, SW & 27 Oct. \\
\hline & 4 & Villaviciosa & $100-180$ & $\mathrm{~S}, \mathrm{SW}, \mathrm{NW}$ & 19 Oct. \\
\hline \multirow[t]{4}{*}{ Vaquera } & 2 & Belmonte de Miranda & $300-330$ & E, NW & 27 Oct. \\
\hline & 2 & Ibias & $600-780$ & E & 22 Oct. \\
\hline & 1 & Salas & 480 & $\mathrm{E}$ & 4 Nov. \\
\hline & 3 & Tineo & $300-440$ & $\mathrm{~N}, \mathrm{~W}, \mathrm{SW}$ & $\begin{array}{c}22- \\
27 \text { Oct. }\end{array}$ \\
\hline \multirow[t]{2}{*}{ Vegamesada } & 3 & Ibias & $560-780$ & $\mathrm{E}, \mathrm{S}$ & 22 Oct. \\
\hline & 1 & Pola de Allande & 420 & E & 27 Oct. \\
\hline \multirow[t]{9}{*}{ Verde } & 4 & Aller & $350-690$ & $\mathrm{E}, \mathrm{N}, \mathrm{NE}$ & 2 Nov. \\
\hline & 1 & Cangas de Onís & 10 & $\mathrm{~N}$ & 28 Oct. \\
\hline & 1 & Grado & 520 & W & 26 Oct. \\
\hline & 1 & Ibias & 560 & $\mathrm{~S}$ & 2 Nov. \\
\hline & 3 & Pola de Allande & $550-690$ & $\mathrm{E}, \mathrm{S}$ & 22 Oct. \\
\hline & 1 & Salas & 400 & $\mathrm{~N}$ & 4 Nov. \\
\hline & 1 & Tapia de Casariego & 200 & $\mathrm{E}$ & 25 Oct. \\
\hline & 1 & Taramundi & 300 & $\mathrm{~N}$ & 26 Oct. \\
\hline & 3 & Teverga & $600-700$ & $\mathrm{~N}, \mathrm{E}$ & 28 Oct. \\
\hline Villaviciosa & 2 & Belmonte de Miranda & 350 & W & 27 Oct.- \\
\hline \multirow[t]{2}{*}{ Vizcaína } & 1 & Caravia Baja & 100 & $\mathrm{~N}$ & 2 Nov. \\
\hline & 1 & Laviana & 570 & $\mathrm{E}$ & 4 Nov. \\
\hline Xidra & 2 & Mieres & 300 & W & 2 Nov. \\
\hline Ximara & 1 & Taramundi & 300 & $\mathrm{~N}$ & \\
\hline \multirow[t]{3}{*}{ Zapatona } & 1 & Oviedo & 280 & $\mathrm{~N}$ & 29 Oct. \\
\hline & 2 & Piloña & $300-330$ & $\mathrm{~N}, \mathrm{E}$ & 28 Oct. \\
\hline & 2 & Siero & $200-220$ & All & 25 Oct.- \\
\hline
\end{tabular}

3 Nov.

${ }^{\text {zS }, ~ E, ~ S E, ~ A l l, ~ W, ~ N W, ~ N E, ~ N, ~ a n d ~ S W ~=~ s o u t h-f a c i n g, ~ e a s t-f a c i n g, ~ s o u t h e a s t-~}$ facing, no predominant aspect, west facing, northwest-facing, northeastfacing, north-facing, and southwest-facing slopes, respectively. 
Table 3. Chestnut cultivars found in Castilla-León, province of León.

\begin{tabular}{lccccc}
\hline \hline Cultivar & $\begin{array}{c}\text { No. } \\
\text { trees }\end{array}$ & Municipality & $\begin{array}{c}\text { Elevation } \\
(\mathrm{m})\end{array}$ & $\begin{array}{c}\text { Aspect } \\
{ }^{2}\end{array}$ & $\begin{array}{c}\text { Harvest } \\
\text { time }\end{array}$ \\
\hline Gallego & 2 & Vega & 850 & All & 13 Oct. \\
Inxerta, Injerta & 1 & Sigüeya & 950 & $\mathrm{~N}$ & 13 Oct. \\
& 5 & Palacios del Sil & 800 & E, SW & --- \\
Marelo & 3 & Villafranca & $650-880$ & All & 13 Oct. \\
Negral & 8 & Médulas & 700 & All & 13 Oct.-5 Nov. \\
& & Pombriego & 440 & W & 13 Oct. \\
Parede & 2 & Villafranca & $540-880$ & N, S & 13 Oct. \\
Rapada & 1 & Vega & 850 & All & 13 Oct. \\
Rapega & 1 & Villafranca & 880 & $\mathrm{~S}$ & 13 Oct. \\
Verdello & 1 & Villafranca & 880 & $\mathrm{~S}$ & 13 Oct. \\
\hline
\end{tabular}

${ }^{\mathrm{z}} \mathrm{All}, \mathrm{N}, \mathrm{E}, \mathrm{SW}, \mathrm{W}$, and S = no predominant aspect, north-facing, east-facing, south-west facing, west-facing, and south-facing slopes, respectively.

Table 4. Chestnut cultivars found in Extremadura; Badajoz and Cáceres provinces.

\begin{tabular}{|c|c|c|c|c|c|}
\hline Cultivar & $\begin{array}{c}\text { No. } \\
\text { trees }\end{array}$ & Municipality & $\begin{array}{c}\text { Elevation } \\
(\mathrm{m})\end{array}$ & Aspect & $\begin{array}{c}\text { Harvest } \\
\text { time }\end{array}$ \\
\hline \multicolumn{6}{|c|}{ Badajoz } \\
\hline Temprana & 2 & Cabeza de la Vaca & 900 & North & \\
\hline Tardía & 3 & Cabeza de la Vaca & 900 & North & \\
\hline \multicolumn{6}{|c|}{ Cáceres } \\
\hline Inxerta & 1 & Guadalupe & 800 & North & 29 Oct. \\
\hline Verata & 3 & Guadalupe & 800 & North & 29 Oct. \\
\hline
\end{tabular}

Table 5. New chestnut cultivars found in Galicia, province of Ourense.

\begin{tabular}{lcccc}
\hline \hline Cultivar & $\begin{array}{c}\text { No. } \\
\text { trees }\end{array}$ & Municipality & $\begin{array}{c}\text { Elevation } \\
(\mathrm{m})\end{array}$ & Aspect $^{2}$ \\
\hline Cruzá & 2 & A Bola & 500 & East \\
& 1 & A Merca & 500 & All \\
De Fecha & 2 & A Merca & 500 & All \\
Negrá & 1 & A Bola & 500 & East \\
Verdeas & 1 & A Merca & 500 & All \\
\hline
\end{tabular}

${ }^{\mathrm{z}} \mathrm{All}=$ no predominant aspect.

nuts has encouraged land owners to rejuvenate the old orchards, top-working them to profitable cultivars. This has happened more in the southern part of Andalucía than in the west.

Influence of altitude. The lowest elevation of sampling in Andalucía was in the west, where chestnut is grown from 470 to $600 \mathrm{~m}$. In southern Andalucía, cultivars are found in more elevated orchards, (650 to $850 \mathrm{~m})$. In Castilla-León, all cultivars were found between 440 and $950 \mathrm{~m}$, and in Extremadura, between 800 and $900 \mathrm{~m}$. Four cultivars where collected from southern Galicia at an elevation of $500 \mathrm{~m}$, including 'De Fecha' and 'Cruzá', which were not reported by Fernández and Pereira (1994). In Asturias, the range of altitudes was from sea level to $900 \mathrm{~m}$, similar to that in Galicia. Of the municipalities with chestnut cultivars that we visited, $39 \%$ were between sea level and $200 \mathrm{~m}, 67 \%$ between 200 and $400 \mathrm{~m}, 45 \%$ between 400 and $600 \mathrm{~m}$, $22 \%$ between 600 and $800 \mathrm{~m}$, and only $6 \%$ between 800 and $1000 \mathrm{~m}$. Therefore, chestnut orchards in Asturias are located mainly between sea level and $600 \mathrm{~m}$. In comparison, the chestnut orchards in Galicia were in more elevated areas, between 400 and $800 \mathrm{~m}$ (Fernández and Pereira, 1993). Over 45\% of municipalities with chestnuts had grafted cul- ripen after 25 Oct. Growers said that late cultivars were predominant in the past, but now harvest after 11 Nov. is very rare.

\section{Conclusions}

In the regions of Andalucía, Asturias, Castilla-León, Galicia, and Extremadura we found chestnut cultivars in 131 municipalities. We have added 45 new cultivars and located examples of most of the cultivars previously reported (Fernández and Pereira, 1993; Fernández-Lamuño, 1984). Fewer than $50 \%$ of the cultivars are widely grown in large numbers in any area. Trees that produced well and had good nuts were shared among growers until they were widespread.

Chestnut cultivars in Spain are grown at elevations from sea level up to $1100 \mathrm{~m}$, but are more frequently planted between 200 and 800 $\mathrm{m}$. Aspect is important in all areas, with northfacing slopes being most suitable for production.

Harvest in Spain begins $\approx 15$ Sept. in Andalucía, followed by Castilla-León and some areas of southeastern Galicia, which produce nuts after 10 Oct., and finishes $\approx 23$ Nov. in Asturias. Most of the cultivars are harvested from 25 Oct. to 10 Nov.

Representatives of these Spanish chestnut cultivars will now be preserved and tested for genetic characteristics in our germplasm repository, located at the Centro de Investigaciones Forestales e Ambientais, Xunta de Galicia, in collaboration with the Univ. of Santiago de Compostela, Spain.

\section{Literature Cited}

Colinas, C. and M. Uscuplic. 1999. Studies on chestnut blight [Chryponectria parasitica (Murr.) Barr] in north-east Spain. Acta Hort. 494:495-500.

in low areas where ink disease (caused by Phytophthora sp.) could be a serious problem.

Aspect. As reported by Fernández and Pereira (1993) and Elorrieta (1949), Spanish chestnut trees grow best on north-facing slopes, especially in areas where drought is frequently a problem, such as in Andalucía and Extremadura. In areas with more abundant moisture, as in Asturias, the orientation of the orchards is not as important.

Harvest time. Most of the chestnuts in Spain are harvested between 26 Oct. and 20 Nov. Harvest begins in southern Andalucía between 15 Sept. and 10 Oct. with the cultivars Pilonga and Temprana (Table 1). Harvest continues in Castilla-León on 13 Oct. (Table $3)$. 'Negral' also is harvested in southern Galicia $\approx 13$ Oct. (Pereira-Lorenzo and Fernández-López, 1997). In Asturias, harvest begins on 19 Oct., with cultivars such as 'Dela Grúa', 'Pelona', 'Valduna', 'Chamberga', 'Miguelina', 'Montesín', 'Negral', and 'Mariana' (Table 2). At this time, some cultivars from western Andalucía also begin to produce nuts (Table 1).

Late or very late cultivars are harvested after 25 Oct. In southern Andalucía, some cultivars, such as 'Tomasa' and 'Rubia Tardía',
Elorrieta, J. 1949. El castaño en España. Min. de Agr., Pesca y Alimentación, Madrid.

Fernández, J. and S. Pereira. 1993. Inventario y distribución de los cultivares tradicionales de castaño (Castanea sativa Mill.) en Galicia. Serie Recursos Naturales, Instit. Nacional de Invest. Agrarias 87.

Fernández-Lamuño, J.A. 1984. Variedades del castaño como árbol frutal en el occidente de Asturias, p. 133-150. In: Xunta de Galicia (ed.). Congreso Intl. del Castaño, Dept. Invest.

Ministerio de Agricultura, Pesca y Alimentación, 1997. Anuario de estadística agraria, p. 526533. Min. de Agr.,Pesca y Alimentación, Madrid.

Pereira-Lorenzo, S. and J. Fernández-López. 1997. Description of 80 cultivars and 36 clonal selections of chestnut (Castanea sativa Mill.) from northwestern Spain. Fruit Var. J. 51:13-27.

Pereira-Lorenzo, S., J. Fernández-López, and J. Moreno-González. 1996a. Variability and grouping of Northern Spanish cultivars of chestnut (Castanea sativa). I. Morphological traits. J. Amer. Soc. Hort. Sci. 121:183-189.

Pereira-Lorenzo, S., J. Fernández-López, and J. Moreno-González. 1996b. Variability and grouping of Northern Spanish cultivars of chestnut (Castanea sativa). II. Isoenzyme traits. J. Amer. Soc. Hort. Sci. 121:190-197. Forestal de Lourizán, Pontevedra, Spain. 